\title{
Rethinking Context-aware Computing to Support Reflective Engagement
}

\author{
A. Baki Kocaballi \\ Australian Institute of Health Innovation \\ Macquarie University \\ baki.kocaballi@mq.edu.au
}

\author{
Claudia Núñez-Pacheco \\ School of Design \\ Universidad Austral de Chile \\ claudia.nunez@uach.cl
}

\begin{abstract}
Context-aware technologies increasingly become more integrated into people's everyday lives, providing adaptive services in seamless ways that make many everyday tasks and activities more practical and automated. However, this seamless automation of services may prevent people from reflecting on the opportunities offered by their surroundings. The perspective proposed in this paper invites designers to rethink the role of context-aware technologies as mediators of humans' capacity to reflectively engage with their surroundings. Drawing upon the design qualities offered by seamful design and New Brutalism movement, the paper offers two ways in which contextaware technologies can support reflective engagement: visibility of technology and visibility through technology.
\end{abstract}

Context-awareness, reflection, engagement, awareness, visibility, space

\section{INTRODUCTION}

Context-aware computing is one of the key application domains supporting ubiquitous computing's main vision of seamless integration of computing technologies operating invisibly at background. A system can be considered contextaware if it can capture, interpret and use contextual information and provide adaptive services according to the current context of use relevant to the user's task (Dey 2001).

Context-awareness can be examined as active or passive (Chen \& Kotz 2000). While applications employing active context-awareness automatically adapt their behaviour and services according to the current context, applications utilizing passive context-awareness merely make the current contextual information available to the user. Active context-awareness with its aim of seamless integration of information and services is more suitable for the main vision of ubiquitous computing, whereas passive context-awareness is usually a less preferable type of awareness due to its constant reliance on humans' input.

In this paper, we argue that passive context-aware technologies with an explicit focus on the visibility of and through technologies may prove useful for increasing humans' awareness of their surrounding environment and improving their capacity to relate to other entities in their environment. First, we will present an alternative understanding of the idea of invisibility in ubiquitous computing and explain how seamful technologies have implemented this idea.
After discussing the benefits of making humans more context-aware, we will introduce two main strategies to support context-aware humans with various examples from the literature.

\section{SEAMFUL TECHNOLOGIES}

In his early seminal work, Weiser described his vision of a promising future enabled by invisible technologies embedded into everyday environment of people: "The most profound technologies are those that disappear. They weave themselves into the fabric of everyday life until they are indistinguishable from it." (Weiser 1991, p.1). His vision of invisible computing has been very influential for shaping the fields of pervasive and ubiquitous computing. However, later, he explained that he is actually supportive of seamful technologies (Weiser 1994). What he meant by invisible was the ways in which technologies are used and operate at the background of our consciousness. Similarly, Tolmie et al. (2002), who proposed 'unremarkable computing' instead of ubiquitous computing, explained that seamless interaction or invisibility should concern the actual use of technologies rather than perceptual qualities of technologies themselves.

A few other researchers (Chalmers \& Galani 2004; Galloway 2008; Rogers 2006) also drew our attention to the limitations and misunderstandings about the idea of invisibility in Ubicomp. Rogers (2006) argued that a new direction for Ubicomp was needed in order to bring back 'excitement of interaction' and to 'augment the human intellect so 
that people can perform ever greater feats, extending their ability to learn, make decisions, reason, create, solve complex problems and generate innovative ideas' (Rogers 2006, p. 8). She opposed to the idea of embedded computing technologies operating at the background and advocated a new approach in which technologies 'causing us to reflect upon and think about our interactions with them' (p. 9). Rogers claims that a shift from 'proactive computing to proactive people' was needed, leading to designing technologies that are not doing things on behalf of people but enabling them to become more actively involved with their everyday activities.

Similarly, seamful design proposed by Chalmers and Galani (2004) and the notion of accounts suggested by Dourish and Button (1998) emphasise the importance of increased visibility of technological systems to support user appropriation and reflective engagement. Seamful design (Chalmers \& Galani 2004) advocates the use of (beautiful) seams in interactive systems: seams can be basically gaps and breaks in functionality, and boundaries between different components or systems. Seamful design deliberately makes the seams visible and encourages system users to appropriate them as a resource for reflection and open-ended engagement.

In their seamful game, Chalmers and Galani (2004) utilized deficiency of technological infrastructure, in this case the varying accuracy of the GPS signal, as a seamful resource for players to develop strategies. Rather than considering the variability of technological infrastructure as something to avoid, prevent or hide, they exposed the seams in their design and used them as a feature of the game. Such seamfulness allows recognition of the roles or working principles of these technologies, which explicitly convey what they do but not how they should be used. In this respect, seamful design also supports user appropriation by making resources publicly available.

Dourish and Button's (1998) notion of 'accounts' also advocates seams in design by suggesting the use of self-explanatory and transparent system components. They developed the notion of accounts in order to deal with the difficulties caused by system abstractions. Button and Dourish claim that 'information hiding' characteristic of interfaces prevents users from perceiving some essential operations of the systems. Users may need such information about system operations especially during breakdowns in the system's functionality. If abstractions of system operations can be made observable, users will be better equipped to deal with any breakdowns. That is, systems may provide more information about their operations.

Seamful design's seams and technomethodology's accounts share a similar concern of explicating material qualities of technologies in a situated way. Both seams and accounts emerge from the interactions between humans, technologies and their respective environments. In this paper, we propose a new perspective involving seamful context-aware technologies to support contextaware humans.

\section{WHY CONTEXT-AWARE HUMANS}

In a conventional context-aware computing system, what is context-aware does not refer to humans but technological systems adapting to the environment. The contextual information, which includes information about humans and other entities in space, is captured by sensors and interpreted by technological systems. The interpretation of the contextual information is often performed in a way that produces a codified context in a format that is accurate, precise, and easy to understand.

Active context-aware systems provide the contextual information for various contextdependent services operating at the background in a seamless manner. However, passive contextaware systems present the contextual information in a neat and clear fashion that support direct and easy communication of the information, which is the only suitable presentation way for some cases such as safety critical situations. Our aim is to characterise another stream of research supporting context-aware humans having more active and reflective relationships with their surrounding environment in everyday situations. We argue that facilitating context-aware humans may prove useful for (i) increasing awareness of some overlooked aspects of space (Falk et al. 1999); (ii) empowering people to re-appropriate technology and space (Kocaballi et al. 2011); (iii) supporting error handling in a situated way (Button \& Dourish 1996); (iv) supporting everyday creativity (Wakkary \& Maestri 2007); and, (v) generating new relations between people, technology, and space (Dunne \& Raby 2013).

\section{FACILITATING CONTEXT-AWARE HUMANS}

In the early 1950s, an influential architectural movement referred to as New Brutalism advocated design qualities that were aligned with the idea of context-aware humans. The founder of this movement, Reyner Banham, proposed three key design qualities: '1. Memorability as an image; 2. Clear exhibition of structure; and 3. Valuation of material as found (Banham 1955, p. 23). In effect, the second and third qualities are associated with the seamful visibility we advocate in this paper. Using Hunstanton School (see Figure 1), as an example, Banham emphasized the importance of clear exhibition of structure. 'One can see what Hunstanton is made of, and how it works, and there is not another thing to see except the play of 


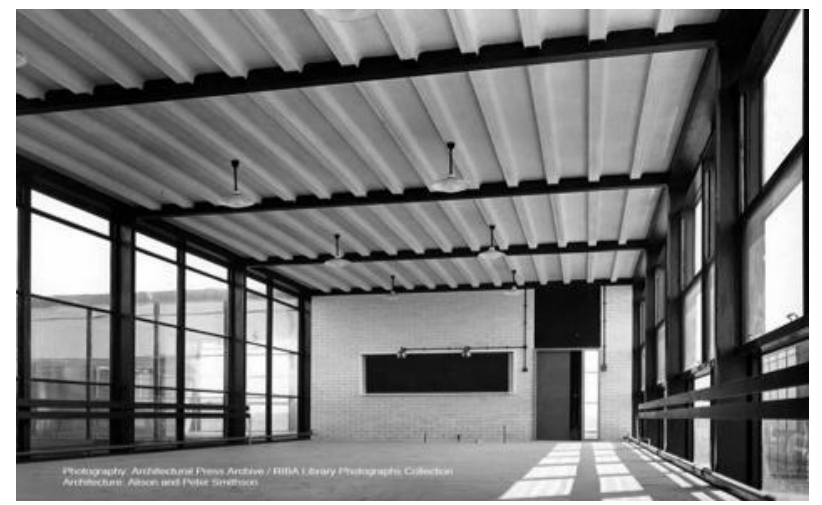

Figure 1. Clear exhibition of structural components in Hunstanton School (Source: RIBA Library Photography Collection)

spaces' (Banham 1955, p. 22). Since no "information hiding" occurs in the "interface", inhabitants of the buildings can relate to the environment in a more resourceful and informed way.

While exhibition of material and structure support people's awareness of their architectural environment, computing technologies can be considered another kind of material that can support the awareness of themselves and other contextual elements in the environment. Drawing upon the design qualities offered by New Brutalism movement, we propose two design qualities to support context-aware humans: visibility of computing technologies, and visibility through computing technologies.

\subsection{Visibility of technology}

In the case of visibility of technology, a particular technology is just responsible for itself: the realities outside of the technology are not dealt with. A technological device or system would make visible its own properties and functionality. Bardzell employs the concept of self-disclosure referring to 'the extent to which the software renders visible ways in which it affects us as subjects' (Bardzell 2010 , p. 1307). She cites the recommendation system of Amazon.com as an example of selfdisclosure. Users of Amazon can select the option - "Don't use for recommendations" - which prevents the system from using the current purchase as an exemplary case to recommend other books. Here, the recommendation system allows users to express themselves to Amazon as 'what kind of subject I want the application to treat me as' (Bardzell 2010, p. 1308). The case of Amazon demonstrates a highly sophisticated mode of visibility, which explicates the way in which machines view users and provide resources for users to configure the machine's perceptions of said users. Here, visibility, in the form of selfdisclosure, facilitates more responsible effects in design.
Another useful example is amplified reality proposed by Falk et al. (1999), as a complement to augmented reality. They differentiate the two in the following way:

While augmented reality is about enhancing our impressions of everyday objects in our surroundings amplified reality is about enhancing the expressions of objects and people in the world ... An amplified object is self-contained in regard to its properties. In practice, this means that the properties are embedded parts of the object. In contrast, augmented reality superimposes virtual properties on an object, which in effect does not change the actual object, but rather how we perceive or experience it. (p. 3)

An example of amplifying expression of people is the Hummingbird (Holmquist et al. 1999), which support mutual awareness between two people frequently visiting the same place. The Hummingbird is a wearable device providing its users with sonic and visual feedback about the existence of other people who might be out of visible range of the wearer of the device. The device amplifies the presence of people in the shared places. In a similar way, Virtual Intimate Object (VIO) (Kaye et al. 2005) amplifies the presence of couples pursuing a long-distance relationship. What is remarkable with $\mathrm{VIO}$ is the provision of an intimate feeling of presence through a minimal visual indication, i.e., just a single led light.

The Canary (DiSalvo et al. 2008) is a device equipped with six sensors: air quality, light, sound, humidity, pressure and temperature. It is a unique piece of technology in terms of its support to two different forms of visibility. While its sensors provide contextual information about the surrounding environment and hence increase the visibility of environment, its semi-transparent and easy-to-open casing allows a closer inspection of interior of the device and consequently enhances the visibility of technology.

Storni connects the quality of visibility with the idea of empowerment-in-use. He argues that restoring sign in de-sign is needed to support user empowerment in use of technologies. This requires making visible a design artefact's properties and 'conditions of its design and its assumptions' (2014, p. 165). He further claims that restoring sign in design enables users to disagree with the 'sign'. Following Chalmers and Galani (2004) and Dourish and Button (1998), he suggests that an explicit focus on making visible seams of design artefacts can facilitate critical engagement of users and empowerment-in-use.

\subsection{Visibility through technology}

In the case of visibility through technology, technological systems mediate and make visible an 
outside situation or entity. The things that made visible are not related to the technological system itself but any other entities constitutive of environment. Such technologies act as mediators of reality. They make actions or artefacts public. In this respect, Robertson draws our attention to importance of the public availability of actions and artefacts to support awareness and collaboration: 'people can only shape their own actions so that they are meaningful in relation to those of others if the ongoing activity is publicly available to each of the participants' (Robertson 2002, p. 303). She notes that perceptibility is key to the public availability.

A simple example of visibility through technology is an augmented reality headset superimposing some information relevant to the targeted object in the environment. The revealed information or property is only available through the technology. Other typical examples include a thermometer that provides a precise value of the temperature of an environment or a microscope that provides magnified images of an entity placed under its lens. Although a thermometer or a microscope support awareness of an entity or environment, they do it in a way that does not invite further interpretation or reflection. We argue that in addition to the visibility an element of ambiguity can facilitate more interpretation and reflection as suggested by Gaver et al. (2003).

Technological infrastructures are another suitable medium to support visibility. Revealing the underlying infrastructure of the systems enable users to appropriate the systems in their own way (Chalmers \& Galani 2004). The aforementioned seamful design approach makes visible seams of technological infrastructure to facilitate the process of accommodation and appropriation. In the seamful game introduced earlier, the weakness of GPS signal was employed as a game feature of deliberate imprecision.

There are also some cases focusing on increasing our awareness of more temporal aspects of everyday life. History Tablecloth (Gaver et al. 2006) indicates the duration of time objects have stood on it. The glowing pattern underneath an object slowly expands according to the time the object stays at the same spot. In this case, technology facilitates an awareness of the history of an artefact in an environment by making visible the duration of time. However, it is important to note that the historical information was not presented in a numerical and precise way; rather, it was displayed in a symbolic and somewhat imprecise manner. This ambiguous visibility provides users with an interpretative flexibility in their meaning making process. It is up to the users what to do with this information. Here the critical point is that by making visible some previously not accessible aspects of the environment this kind of technologies can facilitate new forms of engagements between humans and their environment. The ambiguity involved in presenting the information further supports curiosity and engagement.

A similar case dealing with the history of a space, SoundMirrors (Hallnäs \& Redström 2001) encourages reflection on previous events by recording the sounds in a corridor and playing them back with varying delays. SoundMirrors is part of a series of projects developed in accordance with Slow Technology (Hallnäs \& Redström 2001), which advocates development of technologies to support 'moments of reflection' and 'mental rest' in our increasingly fast-paced environment. Hallnäs and Redström (2001) provide an analogy of a gourmet meal to explain the distinction between fast and slow technology: While a hamburger being fast-food is all about efficiency and mechanics of eating, gourmet meal being slow food is an invitation for reflection on the art of cooking and eating.

Another case with a different modality is the Bench Object (Dunne \& Raby 1995), a smart bench equipped with sensors and heaters, facilitating a peripheral awareness of a stranger in a public space. The bench warms when another person sits on an adjacent bench. The Bench Object allowed people to reflect on their assumptions about intimacy with strangers in public spaces. In this case, the technology facilitates awareness through an unusual and uncomfortable experience as a defamiliarization tactic.

In all these cases, visibility of and through computing technologies as design qualities have facilitated a reflective engagement with the technologies and other entities in space. As demonstrated in the cases, ambiguity, deliberate imprecision, and defamiliarisation were important tactics to support such a reflective engagement.

\section{CONCLUSION}

This paper has introduced an alternative perspective to promote the design of context-aware technologies supporting reflective engagement with the technologies themselves and the surrounding space. In order to do so, two main strategies with some examples were presented: visibility of technology and visibility through technology. It is important to note that our approach does not deny the role of active context-aware technology and its benefits in various everyday scenarios; rather, we advocate for a complementary understanding of awareness inviting humans' active participation in new everyday situations. Ultimately, this paper suggests shifting the focus from context-aware technologies to context-aware humans, inviting us to design for supporting proactive, sentient, and reflective humans. 


\section{REFERENCES}

Banham, R. 1955. The New Brutalism. The Architectural Review, 354-361.

Bardzell, S. 2010. Feminist $\mathrm{HCl}$ : taking stock and outlining an agenda for design. In Proc of $\mathrm{CHI}$ '10. ACM.

Button, G., \& Dourish, P. 1996. Technomethodology: paradoxes and possibilities. In Proc of the SIGCHI conference on Human factors in computing systems. Vancouver, Canada.

Chalmers, M., \& Galani, A. 2004. Seamful interweaving: heterogeneity in the theory and design of interactive systems. In Proc of the conference on Designing interactive systems. Cambridge, MA, USA.

Chen, G. and Kotz, D. (2000). A survey of contextaware mobile computing research. Tech. Report, Dept. of Computer Science, Dartmouth College.

Dey, A.K., 2001. Understanding and using context. Personal \& Ubiquitous Computing, 5(1), pp.4-7.

DiSalvo, C., Nourbakhsh, I., Holstius, D., Akin, A., \& Louw, M. 2008. The Neighborhood Networks project: a case study of critical engagement and creative expression through participatory design. In Proc of the Conference on Participatory Design. Indiana University.

Dourish, P., \& Button, G. 1998. On "Technomethodology": $\quad$ Foundational Relationships between Ethnomethodology and System Design. Human Computer Interaction, 13(4), 395-432.

Dunne, R. and F. Raby 1995. Fields and Thresholds. Architects in Cyberspace, Architectural Design, no. 118, pp. 60-65.

Dunne, A. and F. Raby 2013. Speculative everything: design, fiction, and social dreaming. Cambridge: MIT Press.

Falk, J., Redström, J., \& Björk, S. 1999. Amplifying reality. In Proc of Handheld and ubiquitous computing. Springer Berlin Heidelberg.

Galloway, A. M. 2008. A brief history of the future of urban computing and locative media. (Unpublished doctoral thesis). Carleton University.

Gaver, W., Beaver, J., \& Benford, S. 2003. Ambiguity as a resource for design. Proceedings of the SIGCHI conference on Human factors in computing systems, Ft. Lauderdale, Florida, USA.

Gaver, W., Bowers, J., Boucher, A., Law, A., Pennington, S., \& Villar, N. 2006. The history tablecloth: illuminating domestic activity. In Proc of the conference on Designing Interactive systems (pp. 199-208). ACM.

Hallnäs, L. and J. Redström. 2001. Slow technology, designing for reflection. Personal and ubiquitous computing. 5(3): p. 201-212.

Holmquist, L. E., Falk, J., \& Wigström, J. 1999. Supporting group collaboration with interpersonal awareness devices. Personal Technologies. 3(1-2), 13-21.

Kaye J, Levitt M, Nevins J, Golden J, Schmitt V 2005. Communicating intimacy one bit at a time. Proc of CHI '05. ACM, NewYork, pp 1529-1532

Kocaballi, A.B., Gemeinboeck, P., Saunders, R. and Dong, A., 2011. Towards a relational approach to design process. In Proceedings of ANZASCA.

Robertson, T. 2002. The public availability of actions and artefacts. Computer Supported Cooperative Work. 11(3-4), 299-316.

Rogers, Y. 2006. Moving on from weiser's vision of calm computing: Engaging ubicomp experiences. In UbiComp 2006: Ubiquitous Computing (pp. 404-421). Springer Berlin Heidelberg.

Storni, C. 2014. The problem of de-sign as conjuring: empowerment-in-use and the politics of seams. In Proc of the Participatory Design Conference. ACM.

Tolmie, P., Pycock, J., Diggins, T., MacLean, A. and Karsenty, A. 2002. Unremarkable computing. In Proc CHI '02. ACM.

Wakkary, R., \& Maestri, L. 2007. The resourcefulness of everyday design. In Proc of Creativity \& Cognition.

Weiser, M. 1991. The computer for the 21st century. Scientific American 272(3): 78-89.

Weiser, M. 1994. Creating the invisible interface. ACM Conference on User Interface Software and Technology. Marina del Rey, California, US. 